\title{
Suicide Risk Assessment in Major Depressive Disorder Patients Attending the Regional Institute of Medical Sciences in Imphal, Manipur.
}

\author{
Ching Gou Mann ${ }^{1}$, Md Munnawar S Hussain ${ }^{2}$ \\ 1. Medical officer Churachandpur district hospital, Churachandpur, Manipur, India. \\ ${ }^{2}$ Assistant Professor, Department of Psychiatry, KhajaBandanawaz Institute of Medical Sciences, \\ Kalaburagi, Karnataka, India-585102
}

\begin{abstract}
:
Background: Majority of the clinically depressed persons have suicidal ideation, which is directly related to the severity of depression.

Aims: The study aims to identify the socio-demographic factors for suicidal risk behavior and correlation between suicidal risk behavior and clinical variables of depression as well as with the degree of Major depressive disorder severity. It is a hospital based cross- sectional study conducted in the Department of Psychiatry, Regional Institute of Medical Sciences (RIMS), Imphal.

Materials and methods:50 consecutive cases of attempted suicide attending hospital for a period of lyear were taken up for the study. Consenting Patients fulfilling DSM-IV-TR Criteria for major depressive disorderwere evaluated for socio-demographic profile, precipitating events and mode of attempt. 17-Item Hamilton Rating Scale for depression and Suicide Intent Questionnaire were employed. The data thus obtained was analyzed using SPSS 20.0 windows.

Results: Of the fifty (50) suicide attempters (66\%) were males and. Mean age of the patients is 29.0400, unmarried males (62\%), from joint family (72\%), under- matric (52\%), unemployed (40\%), from urban background (64\%), having poor socio-economic status (42\%) were found to be vulnerable factor for suicide attempt. Most common precipitating factors was stressful problems (20\%), most common method of suicide attempt was by use of organophosphorus poisoning (38\%).MDD severity, highest frequency is found in moderate degree (48\%). Maximum SIQ score is found in the definite communicator (5 or more). Among the suicidal behavior, (42\%) of depressed patients reported suicidal ideation.

Conclusion:Correlation between suicidal behavior \& degree of MDD severity was highly significant $(P<0.01)$.

Keywords:Attempted suicide, socioeconomic status, precipitating factors, mode of suicide attempt.
\end{abstract}

\section{Background}

Suicide is among the most tragic events in human life, causing serious distress among relatives and friends as well as imposing a great economic burden on society as a whole. Recent research shows majority of suicides in the world ( 85 percent) occur in low- and middle -income countries ${ }^{1}$

WHO figures show a suicide takes place somewhere in the world every 40seconds. Suicide rates are highest in Europe's Baltic states, where around 40 people per 100,000 die by suicide each year, second in line is in the Sub-Saharan Africa where 32 people per 100,000 die by suicide each year. The lowest rates are found mainly in Latin America and a few countries in Asia ${ }^{3}$ It is estimated that global annual suicide fatalities could rise to 1.5 million by 2020 . Worldwide, suicide ranks among the three leading causes of death among those aged 15-44years. Suicide attempts are up to 20 times more frequent than completed suicides ${ }^{4}$

Men have a higher rate of completed suicide than women, usually the male to female ratio is approximately 3-4:1 (WHO, 2005) ${ }^{5}$. Although 80 percent of persons who commit suicide are men, the majority of those who make a non-fatal suicide attempts are women between 25 and 44 years of age ${ }^{6}$. Firearms, suffocation, and poison are by far the most common methods of suicide overall. In male's firearms is the most common method of suicide whereas in females poisoning is the commonest method of suicide ${ }^{3}$

.Strictly speaking, suicidal ideation means wanting to take one's own life of thinking about suicide without actually making plans to commit suicide.

According to Skeggattempted suicide as a term is used for episodes where there was at least some suicidal intent, or sometimes without reference to intent.

Previous suicide attempt and current major depression are the two best predictors of future suicide, and the vast majority of suicide attempters/completers come from a population of people with current suicidal ideation, particularly in the presence of untreated major depression ${ }^{6}$. More than one-third of suicide victims have at least one previous suicide attempt, and the first suicide attempt significantly increases the risk of completed 
Title: Suicide Risk Assessment in Major Depressive Disorder Patients attending the Regional...

suicide during the next 10-15 years. This is partly due to the fact that repeated suicide attempters frequently switch their method from non-violent to violent or from non-lethal to lethal methods. ${ }^{7}$ Data on suicide attempts are only available from a few country, they indicate that the number of suicide attempts may be upto 20 times higher than the number of completed suicide. ${ }^{\mathbf{8}}$

Beck \& his colleagues showed in their research that there is a strong relationship between life stress and suicidal behavior. Hopelessness has been found to play a major role in suicidal behavior, and in many cases hopelessness has proven to be a better predictor of suicidal intent than depression, and is believed to mediate the relationship between depression and suicidal behavior. ${ }^{9}$

Suicide risk is greater at certain points in the illness or episode course. In the course of major depressive disorder, suicidality tends to occur early, often before a diagnosis has been made or treatment has begun. ${ }^{10}$ After a suicide attempt, the risk for suicide is also greatest initially, with most suicides occurring in the first year after the attempt. ${ }^{1}$ Risk may also vary with severity of symptoms. For example, higher levels of depression have been associated with increased risk of suicide in at least one study. ${ }^{\mathbf{1 2}}$

In addition, higher levels of suicidal ideation and subjective hopelessness also increase risk for suicide and suicide attempts. ${ }^{13}$ With older adults, for example, milder symptoms may be associated with greater risk than moderate symptoms in younger adults. ${ }^{14}$ Consequently, clinicians should consider the severity of a patient's illness when assessing suicide risk.

\section{Indian Scenario}

According to National Crime Bureau (2001), incidence rate of suicide was 11 per lakh population during $2004 .{ }^{15}$ Suicide is one of the 10 major causes of death in India. India ranks second in number of suicide death. ${ }^{16}$ In 1984, around 50,000 people committed suicide. In 1994, around 90,000 people committed suicide. Number of suicide attempts is around 10 times than that of the number of suicides. Pondicherry is considered to be currently the highest rate area ( 7 times the national rate, i.e. 70 per lakh) followed by West Bengal. $16 \%$ of the people, who commit suicide in these two states, are below 30 years of age. Other high risk areas include Maharashtra, Andhra Pradesh, Karnataka, Tamil Nadu and Kerala. Among the 89,195 cases of suicide 52752 (59\%) were males and $36443(41 \%)$ were females, making males to females ratio $1.45: 1 .{ }^{17}$ The majority of suicides in India are committed by those below the age of 30years. The fact that $71 \%$ of suicides in Indiaare by persons below the age of 44years imposes a huge social, emotional and economic burden on the society. ${ }^{18}$ Suicide rates are nearly equal among young men and women ${ }^{19}$, with a consistently narrow male to female ratio at $1.4: 1$

Statistics show that more Indian women die by suicide than their Western counterparts. Poisoning (36.6\%), hanging $(32.1 \%)$ and self-immolation $(7.9 \%)$ were the most common methods used to commit suicide. ${ }^{18}$ It is estimated that 1 in 60 persons are affected by suicide. It includes both those who have attempted suicide and those who have been affected by the suicide of a close family or friend. Thus, suicide is a major public and mental health problem, which demands urgent action.

Suicide in India is slightly above world rate. Of the half million people reported to die of suicide worldwide every year, 20\% are Indians. ${ }^{20}$ In a study published in The Lancet in June 2012, the estimated number of suicides in India in 2010 was about 187,000.A large proportion of adult suicide deaths were found to occur between the ages of 15 and 29years, especially in women. ${ }^{21}$ The southern states of Kerala, Karnataka, Andhra Pradesh and Tamil Nadu have a suicide rate of greater than 15 while in the Northern States of Punjab, Uttar Pradesh, Bihar and Jammu \& Kashmir, the suicide rate is $<$ than $3 .{ }^{22}$

Early detection of suicidal individuals, accurate diagnosis and effective treatment of psychiatric disorders (especially MDD) and improving a physician's recognition of depression and suicide risk evaluation is a significant component of suicide prevention.

There are few epidemiological studies on suicide and attempted suicide in Manipur. Fimate et al ${ }^{23}$ reported that there has been a steady rise in the incidence of hanging in Manipur with a sudden rise from 1986 and peak incidence in 1988. The high incidence is found among younger age group and may also be indirectly related to the high incidence of drug addiction in Manipur.

In our society, suicide has become an increasing public health problem. Despite suicide being closely associated with major depressive disorder, very little research work has been undertaken in Manipur. As a result it will be worth studying on the subjects of suicidal risk assessment in major depressive patients. The findings will help in carrying out effective preventing measures to prevent subsequent fatal suicide acts.

\section{Aims And Objectives}

The aims and objects of the study were: 1.To study the socio-demographic factors for suicidal risk behavior in patients with major depressive disorder. 2. To study the correlation between suicidal risk behavior and clinical variables of depression. 3. To study the correlation between degree of MDD Severity and Suicidal risk behavior. 


\section{Materials And Methods}

The study is a hospital based cross- sectional study. The study was conducted in the Department of Psychiatry, Regional Institute of Medical Sciences (RIMS), Lamphelpat.The duration of study is 2(two) years. Data was collected for one year (1st Nov 2011- $31^{\text {st }}$ Oct 2012). 50 consecutive patients both attending the psychiatric Out Patient Department were taken up for study. The study received the ethical approval from the institutional review board.

\section{inclusion criteria:}

1. Patients between 18 years and above who has given the consent.

2. Patients (both inpatients and out patients) fulfilling the DSM-IV-TR Criteria for major depressive disorder.

3. Both male and female sexes were included for the study.

\section{Exclusion Criteria:}

1. Those patients or relatives who are unable or unwilling to give informed consent.

2. Patients with current substance or alcohol abuse and neurological illness.

3. Patients with co-morbid psychiatric disorder.

4. Patients without a reliable informant.

\section{Assessment tools:}

1. A Semi-structured proforma designed for this study

This proforma includes socio-demographic details like age, sex, religion, marital status, domicile, occupation, education, income, and family type.

2. Diagnosis and Statistical Manual of Mental Disorder (DSM-IV-TR Criteria). American Psychiatric Association, 2000.Based on the clinical assessment diagnosis was made according to (DSM-1V-TR Criteria) clinical descriptions and diagnostic guidelines.

3. Suicidal Intent Questionnaire (Gupta SC ET al ${ }^{38}$ ): to measure the suicidal intent

4. The 17-Item Hamilton Rating Scale for Depression (HAM-D) to measure the severity of depressive symptoms in individuals

Procedure:The patients and their care givers were explained about the procedure and the purpose of the study and those willing to participate and give written consent were enrolled in the study and their sociodemographic data were recorded in a Semi-structured proforma.Patients fulfilling DSM-IV-TR Criteria for major depressive disorder was taken up for study and were administered the 17-Item Hamilton Rating Scale for depression to measure the severity of depression.

Suicidal Intent Questionnaire was employed and the total score was taken to assess the presence of suicidal ideation and its intensity or severity. After meticulous workup with the above procedures, the collected data were tabulated and assessed for the correlation between suicidal risk behavior and clinical variables of depression.

\section{Results}

During the study period of 1year, 50 patients with suicidal attempts who were attending the psychiatric OPD as well as admitted in the psychiatric ward of RIMS were assessed.

\section{I). Sociodemographic profile:}

It is observed that the age distribution in the study sample ranges 17 to 56 years (mean age $=29.04$ and $\mathrm{SD}=8.44$ ) which shows a considerable increase in suicide attempt at the age group of 21-30 years with 46 percent, and at the age group of 31-40 years with 26 percent. The Gender distribution of the sample revealed a male preponderance $(n=33 ; 66 \%)$ compared to female $(n=17 ; 34 \%)$

Majority of the patients, were single unmarried( $n=31 ; 62 \%)$, under matric $(\mathrm{n}=26 ; 52 \%)$, unemployed( $\mathrm{n}=20 ; 40 \%)$ or students( $\mathrm{n}=10 ; 20 \%)$, having a majority of family income in the range of Rs 10,000 or less $(n=21 ; 42 \%)$ mostly belonging to Hindu religion $(n=33 ; 66 \%)$, joint $(n=36 ; 72 \%)$ or nuclear family $(\mathrm{n}=14 ; 28 \%)$,it is also observed that most of the patients are from urban background(n=32;64\%)as compared to rural $(\mathrm{n}=18 ; 36 \%)$.

\section{ii) Clinical profile:}


Title: Suicide Risk Assessment in Major Depressive Disorder Patients attending the Regional...

a)According to MDD severity: Frequencies of MDD severity is highest in the moderate group $(n=24 ; 48 \%)$, followed by severe group $(n=13 ; 26 \%)$ mild group $(n=6 ; 12 \%)$, very severe $(n=4 ; 8 \%) \&$ normal group $(n=3 ; 6 \%)$ respectively.

b) According to SIQ Score: Frequencies of SIQ Score is highest in definite communicator $(\mathrm{n}=29 ; 58 \%)$, followed by partial communicator $(n=13 ; 26 \%)$, and non-communicator $(n=8 ; 16 \%)$

C) According to Suicidal behavior: It is observed that among the subjects with suicidal behavior, majority of the subjects is found to have Suicidal ideation $(\mathrm{n}=21 ; 42 \%)$, followed by suicidal attempt $(\mathrm{n}=14 ; 28 \%)$, suicidal threat $(n=8 ; 16 \%)$, whereas no history of suicide attempt is found in $(n=7 ; 14 \%)$

d)According to precipitating factors: the precipitating factors for suicidal risk behavior are stressful problems $(n=10 ; 20 \%)$, failure in love $(n=9 ; 18 \%)$, low self-esteem \& marital disharmony $(n=6 ; 12 \%)$ respectively, quarrelling with parents( $\mathrm{n}=5 ; 10 \%)$,financial difficulties \& loss of near ones $(\mathrm{n}=4 ; 8 \%)$,conflict with family members \& current illness related $(n=3 ; 6 \%)$

e) According to Mode of suicidal attempt: Most common method used were Organophosphorous poisoning $(n=5 ; 10 \%)$, followed by kerosene poisoning $(n=3 ; 6 \%)$.Patient also used other less common violent methods i.e. burnt $(n=1 ; 2 \%)$; cut injury \& hanging $(n=2 ; 4 \%)$ respectively.

iii) Relation between Suicidal Behavior and Socio-economic factors with respect to

A) Age: Suicide risk behavior is higher in the age group 21-30years $(n=23)$ where number of suicidal attempt is $(\mathrm{n}=9)$, suicidal ideation $(\mathrm{n}=11)$ \& suicidal threat is $(\mathrm{n}=3)$.

B) Sex: Among the males $(n=33)$, it is observed that suicidal attempt is present in $(n=7)$, suicidal ideation $(\mathrm{n}=13)$, suicidal threat $(\mathrm{n}=7)$, and no suicidal attempt is $(\mathrm{n}=6)$

c) Religion: Among the Hindus $(n=33)$, it is found no suicidal attempt in $(n=6)$, suicidal attempt in $(n=10)$, suicidal ideation $(n=11)$, and suicidal threat in $(n=6)$ cases.

d) Marital status: Among the unmarried patients $(n=31$ no suicidal attempt is present in $(n=2)$ cases, suicidal attempt in $(n=10)$, suicidal ideation $(n=13)$, and suicidal threat in $(n=6)$ cases.

E) Locality: Patients residing in Urban areas $(n=32)$ are more involved in suicidal risk behavior as compared to those residing in rural areas $(n=18)$. No suicidal attempt is found in $(n=6)$ cases, suicide attempt in $(n=8)$, suicidal ideation $(n=15)$ \& suicidal threat $(n=3)$

iii) Relation between Suicidal Behavior and Clinical Variables of Depression with respect to

A) Feeling guilt: Among $(n=12)$ cases having symptoms of feeling guilt, no suicidal attempt is present in $(n=2)$ cases, suicidal attempt in $(n=6)$, suicidal ideation in $(n=3)$ \& suicidal threat in $(n=1)$ cases.

B) Suicide: Among $(n=42)$ cases having suicidal symptoms, suicidal attempt is found in $(n=14)$, suicidal ideation in $(n=21)$ and suicidal threat in $(n=7)$ cases.

C) Insomnia Early: Among $(n=35)$ patients having symptoms of Insomnia Early, no suicidal attempt is present in $(n=3)$, suicidal attempt $(n=11)$, suicidal ideation $(n=15)$, suicidal threat $(n=6)$,

D) Insomnia Middle: It is found that $(n=14)$ number of patients having insomnia middle symptoms, were found to have no suicide attempt in $(n=1)$ patients, suicidal attempt in $(n=5)$, and suicidal ideation in $(n=8)$ patients.

E) Insomnia Late: $(n=27)$ number of patients having insomnia late symptoms were found to have no suicide attempt in $(n=3)$, suicidal attempt in $(n=9)$, suicidal ideation in $(n=10)$ \& suicidal threat in $(n=5)$ patients.

F) Work activities: Among $(\mathrm{n}=49)$ cases having work activities symptom, no suicidal attempt is present in $(n=6)$ cases, suicidal attempt $(n=14)$, suicidal ideation in $(n=21), \&$ suicidal threat in $(n=8)$ cases

G)Motor Retardation: It is observed that in $(n=2)$ cases having motor retardation symptoms, suicidal attempt is present in $(n=2)$ cases

H)Agitation: Out of $(n=30)$ cases having symptoms of agitation, $(n=12)$ cases are having suicide attempt, $(\mathrm{n}=12)$ suicidal ideation, and $(\mathrm{n}=6)$ cases having suicidal threat.

I)Anxiety Psychic: Among $n=29$ patients having anxiety psychic symptom, suicidal attempt is present in $(n=9)$ cases, suicidal ideation in $(n=13)$, and suicidal threat in $(n=7)$ cases.

J)Anxiety Somatic: It is observed that $(n=37)$ cases having anxiety somatic symptoms were found to have no suicide attempt in $(n=6)$ cases, suicide attempt in $(n=7)$, suicidal ideation in $(n=21)$ and suicidal threat in $(n=3)$ cases.

K) Somatic Symptoms-Gastrointestinal: $(n=45)$ number of cases having somatic symptom Gastrointestinal were found to have no suicide attempt in $(n=6)$ cases, suicidal attempt in $(n=11)$, suicidal ideation in $(n=20)$, and suicidal threat in $(n=8)$ cases.

L) Somatic Symptoms- General: Among $(n=34)$ cases having somatic symptoms General, $(n=3)$ cases are having no attempt, $(\mathrm{n}=7)$ suicidal attempt, $(\mathrm{n}=17)$ suicidal ideation, and $(\mathrm{n}=7)$ suicidal threat.

M) Genital Symptom: $(n=11)$ cases having Genital symptom presents with no suicide attempt were $(n=1)$ cases, suicidal attempt in $(n=1)$, suicidal ideation in $(n=8)$, and suicidal threat in $(n=1)$

N) Hypochondriasis: In $(n=32)$ cases of Hypochondriasis symptoms, no suicide attempt is present in $(n=5)$ cases, suicide attempt in $(n=7)$, suicidal ideation in $(n=14)$ and suicidal threat in $(n=6)$ cases. 
Title: Suicide Risk Assessment in Major Depressive Disorder Patients attending the Regional...

O) Weight loss: Among ( $n=29)$ cases having symptoms of weight loss, were found to have suicidal attempt in $(\mathrm{n}=12)$ cases, suicidal ideation in $(\mathrm{n}=12)$, and suicidal threat in $(\mathrm{n}=5)$ cases.

P) Insight: Out of $(\mathrm{n}=45)$ number of cases having Insight, cases having no suicidal attempt were $(\mathrm{n}=7)$, suicidal attempt $(n=12)$, suicidal ideation $(n=20)$, and suicidal threat in $(n=8)$.

\section{IV) Relation between MDD Severity with respect to SIQ Score.}

As depicted in (Table: 1) it is found that SIQ Score is highest in the Definite communicator group $(n=29)$, followed by Partial communicator group $(n=13)$, and least in the non-communicator group $(n=8)$ whereas MDD Severity is highest in the moderate degree $(n=24)$ followed by severe degree $(n=13)$, mild degree $(n=6)$, very severe $(n=4)$ and normal $(n=3)$. This finding shows that SIQ Score is found to increase with increase with increase of MDD Severity.

Table: 1

\begin{tabular}{|l|l|l|l|l|l|l|l|}
\hline \multirow{2}{*}{ SIQ score } & \multicolumn{2}{l|}{ MDD SEVERITY MODE } & Total \\
\cline { 2 - 8 } \multicolumn{2}{|c|}{} & Normal & Mild & Moderate & Severe & $\begin{array}{l}\text { Very } \\
\text { severe }\end{array}$ & \\
\hline & & & & 0 & 0 & 0 & 8 \\
& Non-Communicator (0-1) & 3 & 5 & 0 & 2 & 0 & 13 \\
\hline Partial Communicator (2-4) & 0 & 0 & 11 & 11 & 4 & 29 \\
\hline $\begin{array}{l}\text { Definite Communicator (5 or } \\
\text { more) }\end{array}$ & 0 & 1 & 13 & 13 & 4 & 50 \\
\hline Total & 3 & 6 & 24 & & & \\
\hline
\end{tabular}

\begin{tabular}{|l|l|l|l|l|}
\hline Chi-Square Tests & Value & $\mathrm{df}$ & $\begin{array}{l}\text { Asymp. Sig. (2- } \\
\text { sided) }\end{array}$ & P-value \\
\hline & 50.739 & 8 & 0.000 & $\mathrm{P}<0.01$ \\
\hline Pearson Chi-Square & 50 & & & \\
\hline No. of Valid Cases & & & \\
\hline
\end{tabular}

\section{V) Relation between MDD Severity and Suicidal risk behavior}

It is observed in (Table: 2$)$ that maximum number of suicidal ideation $(n=15)$ were having moderate of MDD Severity, $(n=8)$ suicidal attempts were having severe degree of MDD Severity, $(n=5)$ suicidal threat were in moderate degree of MDD severity, no attempt $(n=4)$ in mild degree of MDD severity. It is observed that suicidal risk behavior increases with increase of MDD Severity.

Table: 2.

\begin{tabular}{|l|l|l|l|l|l|l|l|}
\hline \multirow{2}{*}{ Suicidal risk behavior } & \multicolumn{2}{l|}{ MDD SEVERITY MODE } & \multirow{2}{*}{ Total } \\
\cline { 2 - 7 } \multicolumn{2}{|l|}{} & Normal & Mild & Moderate & Severe & Very severe & \\
\hline \multirow{4}{*}{ No attempt } & 3 & 4 & 0 & 0 & 0 & 7 \\
\cline { 2 - 7 } & Suicidal attempt & 0 & 0 & 4 & 8 & 2 & 14 \\
\cline { 2 - 7 } & Suicidal ideation & 0 & 1 & 15 & 3 & 2 & 21 \\
\cline { 2 - 7 } & Suicidal threat & 0 & 1 & 5 & 2 & 0 & 8 \\
\hline Total & 3 & 6 & 24 & 13 & 4 & 50 \\
\hline
\end{tabular}

\begin{tabular}{|l|l|l|l|l|}
\hline Chi-Square Tests & Value & Df & Asymp. Sig. (2-sided) & P-value \\
\hline Pearson Chi-Square & 50.234 & 12 & 0.000 & $\mathrm{P}<0.01$ \\
\hline No. of Valid Cases & 50 & & & \\
\hline
\end{tabular}

\section{A. Socio-demographic characteristics}

\section{Discussions}

1)Age:In this study majority of the patients with suicidal risk behavior were in the age group of 21-30 years (46\%). This is comparable to Indian studies which revealed the vulnerable age group being those between the ages of 18 to 30 years. $^{\mathbf{2 5}, \mathbf{2 6}}$ this may be due to biological vulnerability, insecurity about the future, unemployment, problems of drug addiction, academic failure, marital disharmony and emotional problem.

2)Sex:Males form the majority of the study sample consisting of $(66 \%)$ compared to females (34\%) which is consistent with other Indian study who reported males $(61.3 \%)$ and females $(38.7 \%) .{ }^{27}$ This gender differences can be explained by a number of factors. Men who are depressed are less likely than women to seek help or treatment and are more likely to have comorbid alcohol or substance abuse problems which places men at higher risk. In contrast, women tend to be less impulsive, have more social support, and have lower rates of comorbid alcohol and substance use disorder, all of which have a protective effect. However, this is at variance with Western study wherein majority of attempters were females. ${ }^{\mathbf{2 8}}$ 
Title: Suicide Risk Assessment in Major Depressive Disorder Patients attending the Regional...

3. Religion:It is found that (66\%) are Hindu, (20\%) are Christian and (14\%) are Muslims. Similar finding was found in previous studies which reported Hindus being (76\%) and others (24\%). ${ }^{29}$ This significantly high percentage of Hindu may be due to dominated Hindu domiciles of the place

4. Marital status:The percentage of unmarried (62\%) was significantly higher in the study population. Married were (32\%), divorce and widows constituted only $4 \%$ and $2 \%$ respectively. Gupta S.C et al found unmarried (62\%), married (32\%), divorced and widowed $(6 \%)$ which is comparable with the present finding. ${ }^{29}$ this high percentage in unmarried individuals may be due to the fact that unmarried depressed individuals have decreased social integration and lack of sense of responsibility toward others so they were more vulnerable to suicide risk behavior.

5. Residence: $(n=32 ; 64 \%)$ were from urban background and $(n=18 ; 36 \%)$ were from rural background. The fact that predominance cases were from urban backgrounds perhaps reflects the transition of the Indian society and the stress associated with it. This higher percentage of patients from urban area found in this study may presumably be due to awareness of the need to seek medical help which is favored by the easy availability of the means of transportation in the urban set up. Lack of the same may be the reason for lower number of patients from rural areas .This finding is consistent with other Indian studies ${ }^{25,29 .}$

6. Occupation: Unemployed individuals made up the maximum percentage $(40 \%)$ whereas $(20 \%)$ were students, $(16 \%)$ were business, and $(14 \% \& 10 \%)$ were employed and housewife respectively. In a study on employment status in a sample of $(n=137)$ of suicide attempters and reported that $(46 \%)$ were unemployed, $15.3 \%)$ were employed as farmer, $(26.3 \%)$ were laborer, $(1.5 \%)$ were drivers, $(7.3 \%)$ were housewife and $(3 \%)$ were students. Those unemployed were significantly over represented in the suicide attempters than in controls. ${ }^{31}$ other studies also report similar findings. ${ }^{6,30}$

7. Family income/Financial status: It is observed that majority of the cases belongs to low socioeconomic status $(42 \%)$ then followed by lower middle (34\%), upper middle (14\%) and the least was found in upper group (10\%). Lower socio economic backgrounds had elevated risk for suicidal behavior is in agreement with the studies reported by previous authors ${ }^{6,32,27,33}$.

8. Educational qualification: It is observed that under matriculate group (52\%) constitutes majority of the total sample followed by undergraduate $(28 \%)$ and graduate $(14 \%) \&$ illiterate $(6 \%)$, indicating a higher rate towards the lower educational status. Result suggest that lower education may predispose a person to suicide risk behavior through stress, poverty, unemployment, and substance related disorder .Similar observation is also reported by earlier studies. ${ }^{6,31,33,27}$

9. Type of family: This study shows that more number of the subjects belonged to joint families (72\%) as compared to nuclear families (28\%). In India the joint family concept still exists and people are living in joint rather than in the nuclear family set ups. This high frequency among the joint family is probably related to the stress within the joint family which is very common. This may be the contributory factor as to why such a significant high percentage of patients come from joint family. More number of joint family as found in this study is in consistent with previous studies. ${ }^{34,35}$

B.Classification according to MDD Severity: The frequency of MDD severity is highest in moderate group $24(48 \%)$, followed by severe group $13(26 \%), 6(12 \%)$ in mild group, and $4(8 \%) \& 3(6 \%)$ in very severe group and normal group respectively. This results shows higher severity of depression is a risk factor for suicidal behavior which is similar with that reported by earlier study. ${ }^{36}$

C. Classification according to SIQ Score: Majority of the study sample had severe suicidal intent (58\%) followed by moderate suicidal intent (26\%) and low suicidal intent (16\%). The frequencies of SIQ Score is highest in definite communicator $(58 \%)$, followed by partial communicator $(26 \%)$, and non-communicator $(16 \%)$. Similar findings that suicide intent is more with higher SIQ Score of the patients is suggestive of higher suicide intent is also reported by earlier studies. ${ }^{37}$

D. Classification according to Suicidal Behavior: It is observed that among the subjects with suicidal behavior, majority of the subjects were found to have suicidal ideation (42\%), followed by suicidal attempt (28\%), and suicidal threat $(16 \%)$. Similarly earlier studies reported that all significant suicidal risk factors were more strongly related to ideation than to progression from ideation to plan or an attempt ${ }^{12}$.In a study on adolescent students found the prevalence of suicide risk behavior is quite high with almost $16 \%$ having suicide ideation and $5 \%$ having attempted suicide. ${ }^{38}$

E. Precipitating factor: The common precipitating factors for suicidal behavior are stressful problems (20\%), failure in love (18\%), low self-esteem \& marital disharmony (12\%), quarrelling with parents/siblings 
Title: Suicide Risk Assessment in Major Depressive Disorder Patients attending the Regional...

(10\%), financial difficulties \& loss of near ones (8\%), conflict with family members \& substance abuse $(6 \%)$ which is similar with that reported by other studies $\mathbf{3 9 , 4 0 , 4 1}$.

F. Method of suicide attempt:Organophosphorous poisoning was the most frequent method employed for attempting suicide (10\%).Patient also used other less common violent methods, Kerosene poisoning $(6 \%)$ burnt $(2 \%)$, cut injury \& hanging $(2 \%)$ respectively .Easy availability and lack of safety precaution during storage at home make Organophosphorous agents as the first choice employed for attempting suicide by the attempters.In an Indian study on a total of 504 suicidal attempters admitted to emergency wards, the frequent mode of attempting suicide was by organophosphorus compounds $(66.3 \%)$ followed by over dosage of tablets $(17.8 \%){ }^{27,39,35}$

\section{G. Relation Between Mdd Severity And Suicidal Intent/Behavior.}

1. MDD severity mode w. $r$.t SIQ scores: The correlation between MDD severity mode and SIQ Score is statistically significant $(\mathrm{P}<0.01)$ which reveal that SIQ score increases with increase in the degree of MDD severity. Similarly, positive correlation $(r=48, p<0.05)$ between scores of depression and SIQ score was observed in other study. ${ }^{42}$

2. MDD severity mode with suicidal behavior: Degree of MDD severity have been shown to correlate with suicidal behavior of the patients $(\mathrm{P}<0.01)$. We observed that suicidal risk behavior increases with increase in the degree of MDD severity. This finding is comparable with other study which confirmed the association between severity of depression \& suicidal behavior ${ }^{43}$.

\section{Conclusion}

This study shows that depressed young patients in the age group 21-30 years, male, unmarried, unemployed, joint family,from low socio-economic class having stressful problems, with agitation, anxiety and having high score on SIQ and high degree of MDD severity were more vulnerable to suicide risk behavior. This study also identifies suicidal ideation as the commonest risk factor for suicidal behavior. However higher scores on subjective depression, higher scores on SIQ were reported by the ideators and attempters. Patient with high suicide intent score should be referred to mental health specialist for urgent mental health assessment, because they are at high risk of completed suicide. The correlations observed between suicidal behaviors with clinical variables of depression suggest that timely recognition of suicidal behavior in depressed patients is an equally crucial factor in the implementation of effective preventive policies. This study reveals that suicidal risk behavior among the vulnerable groups needs to be evaluated and managed effectively in order to reduce the rates. This study also stresses the importance for professionals like general practitioners to be trained to identify suicidal risk behaviors so as to facilitate referral and effective management. Indian studies in this area are very few and there is a great need to conduct research in this area. Since the present study is merely confined to one general hospital (RIMS, Imphal), it is not possible to generalize these findings to all population. As such, community based studies in the field, which can reveal a true picture of suicide risk behavior in major depressive patients should be undertaken. The present study suggest the need for early, prompt diagnosis and treatment of major depression so as to prevent such suicidal behavior.

\section{References}

[1]. Krug EG, Dahlberg LL, Mercy JA et al. World Report on violence and Health. World Health Organization, Geneva; 2002. Staff (2002)."Self-directedviolence"

http://www.who.int/entity/violence_injury_prevention/violence/world_report/factsheets/en/selfdire(PDF).WorldHealth rganization. Retrieved 2006-04-11

[2]. Staff(February16, 2006). "SUPRE" http://www.who.int/mental health/prevention/suicide/supresuicideprevent/en/index.html).WHO sites: Mental Health. World Health Organization. Retrieved 2006-04-11.

[3]. WorldHealthOrganization.Countryreportsandchartsweb

page.www.who.int/mental_health/prevention/suicide/country_reports/en/index.html. Accessed January 2005.

[4]. Fawcett J, Clark DC, Busch KA. Assessing and treating the patient at risk for suicide. 1993; 23:244-55.

[5]. Skegg K. Self-harm. Lancet 2005; 366: 1471-1483

[6]. IsometsaET andLonnqvist JK. Suicide attempts preceding completed suicide. British Journal of Psychiatry 1998; 173:531-33

[7]. World Health Organization (WHO). The ICD-10 classification of Mental and Behavioral disorders, clinical descriptions and diagnostic guidelines: World Health Organization, Geneva, 1992

[8]. Beck AT, Brown G, Berchick RJ, Steward BL, Steer RA. Relationship between hopelessness and ultimate suicide: a replication with psychiatric outpatients. American Journal of Psychiatry 1990; 147:190-195.

[9]. Malone KM, Haas GL, Sweeney JA, Mann JJ: Major depression and the risk of attempted suicide. J Affect Disord 1995; 34:173185

[10]. Nordstrom P, Samuelsson M, Asberg M: Survival analysis of suicide risk after attempted suicide. ActaPsychiatrScand 1995; 91:336-340

[11]. Hagnell O, Rorsman B: Suicide in the Lundby study: a comparative investigation of clinical aspects. Neuropsychobiology 1979; 5:61-73 
Title: Suicide Risk Assessment in Major Depressive Disorder Patients attending the Regional...

[12]. Mann JJ, Waternaux C, Haas GL, Malone KM: Toward a clinical model of suicidal behavior in psychiatric patients. Am J Psychiatry 1999; 156:181-189

[13]. Conwell Y, Lyness JM, Duberstein P, Cox C, Seidlitz L, DiGiorgio A, Caine ED: Completed suicide among older patients in primary care practices: a controlled study. J Am GeriatrSoc 2000; 48:23-29

[14]. Gururaj G. Injuries in India: A national perspective. In NCMH Background Papers, Burden of Disease in India, National Commission on Macroeconomics and Health, Ministry of Health and Family Welfare, Government of India. New Delhi, September 2005:334, 338, 340 .

[15]. Gelder M, Mayou R, Cowen P. Suicide and deliberate self-harm. In: Shorter Oxford Textbook of Psychiatry. Oxford University Press:2001:507-9, 511-12

[16]. Unni KES. Human self destructive behavior, Text Book of Postgraduate Psychiatry; Vyas JN and Ahuja N: Jaypee Brothers Medical Publishers (P) Ltd., New Delhi, 2nd edition, 1999; 2:526-56

[17]. Ministry of Home Affairs. Accidental Deaths and Suicides in India. National Crime Research Bureau, Government of India 2005.

[18]. Mayer P and Ziaian T. Suicide gender and age variations in India. Are women in India society protected from suicide? Crisis 2002; 23:98-103.

[19]. Singh A.R., Singh S.A, Preface, towards a suicide free society: identifying suicide prevention as public health policy, Mens Sana Monographs, II 2003; 2:0, 1

[20]. Patel, V.; Ramasundarahettige, C.; Vijayakumar, L.; Thakur, $\quad$ J.S.; Gajalakshmi, V.; Gururaj, G.; Suraweera, W.; Jha, P. (2012). Suicide mortality in India: A nationally representative survey". The Lancet 379 (9834): 2343.

[21]. Vijaykumar L. Suicide and its prevention: The urgent need in India, Indian J Psychiatry 2007; 49:81-84

[22]. Fimate L, Singh AM and Singh TB. Profile of hanging in Manipur. Journal of Medical Society 1993; 16:29-35.

[23]. Gupta S.C et al. Development of a suicidal intent questionnaire. Indian Journal of Psychiatry 1983; 25(1):57-62

[24]. Narang R, Mishra BP, Mohan N. Attempted suicide in Ludhiana. Indian Journal of Psychiatry 2000; 42: 83-7.

[25]. Nandi DN, Banarjee G, Boral GC. Suicide in West Bengal- A century apart. Indian J Psychiatry. 1978; 20: 155-60.

[26]. Nagendra Gouda MR and Sambaji M Rao. Factors related to attempted suicide in Davanagere. Indian Journal of Community Medicine. 2008 January; 33(1):15-18.

[27]. Matthew K. Nock, Ronald C. Kessler. Prevalence of and risk factors for suicide attempts versus suicide gestures: Analysis of the National Co- morbidity Survey. Journal of Abnormal Psychology 2006; 115 (3): 616-623

[28]. Gupta, S.C and Singh, H. Psychiatric illness in suicide attempters. Indian J Psychiatry 1981, 23(1), 69-74.

[29]. Pablo RY and Lamarre CJ: Parasuicides in a general hospital psychiatry unit: Their demographic and clinical characteristics, General Hospital Psychiatry 1986; 8: 279-286

[30]. Srivastava MK, Sahoo RN, Ghotekar LH, Dutta S, Danabalan M, Dutta TK and Das AK: Risk factors associated with attempted suicide: a case control study, Indian Journal of Psychiatry 2004; 46 (1):33-38

[31]. Gupta S.C, Harjeet Singh and J.K.Trivadi: Evaluation of suicidal risk in depressives and schizophrenics: A 2-year follow up study. Indian Journal of Psychiatry 1992: 34(4), 298-310

[32]. Beautrais A.L., Peter R.Joyce and Roger T. Mulder: Youth suicide attempts: a social and demographic profile. Australian and New Zealand Journal of Psychiatry 1998; 32: 349- 357.

[33]. PirduttBansal, Amit Gupta, Rajiv Kumar. The psychopathology and the sociodemographic determinants of attempted suicide patients. Journal of clinical and diagnostic research 2011 Oct 5(5): 917-920.

[34]. Upadhyaya SK, Sudhir G, Sharma A, Joshi A: A study of psycho-socio-demographic variables of suicide attempters in hilly areas of Uttarakhand 2012; Vol 24.No 3

[35]. Malone KM, Oquendo KM, Haas GL, Ellis SP, Li S, Mann JJ: Protective factors against suicidal acts in major depression: Reasons for living. Am J Psychiatry 2000; 157: 1084-1088.

[36]. Anand R, Trivedi JK, Gupta SC. Suicidal communication in psychiatric patients. Indian J Psychiatry.1983; 25:121-8

[37]. Sharma RC: Attempted suicide in Himachal Pradesh, Indian Journal of Psychiatry 1998; 40(1):50-54

[38]. Ponnudurai R, Jayakar J, Saraswathy M. Attempted suicides in Madras. Indian Journal of Psychiatry 1986, 28(1):59-62

[39]. Latha KS, Bhat SM and D'Souza P: Suicide attempters in a general hospital unit in India: their socio-demographic and clinical profile-emphasis on cross-cultural aspects, ActaPsychiatricaScandinavica 1996; 94: 26-30

[40]. SubhadipBharati, SharmilaMallik, PratyayPratimDatta, AsishMukhopadhyay, DebalinaDatta, Sahina Haq: Socio-demographic profile and suicidal intent of attempted suicide cases. A hospital based study in West Bengal, India 2013; 3(2).

[41]. Khalid. A., Lal, N., Trivedi. J.K., Dalal.P.K\&Akhtar, A. Suicidal intent communication in depressed patients. Indian Journal of Behavioral Sciences 1996.6.2, 1997.7.1.26-30

[42]. Srivastava S, Kulshreshtha N. Expression of suicidal intent in depressives. Indian J Psychiatry 2000; 42: 184-7

[43]. Beck AT, Steer RA, Kovacs M, Garrison B: Hopelessness and eventual suicide: a s10-year prospective study of patients hospitalized with suicidal ideation. American J Psychiatry 1985; 142:559-563 